\title{
Localized Data Dissemination in Vehicular Sensing Networks
}

\author{
Ling-Jyh Chen, Ying-Yu Chen \\ Institute of Information Science \\ Academia Sinica \\ $\{$ cclljj, ciy\}@iis.sinica.edu.tw
}

\author{
Kun-chan Lan, Chien-Ming Chou \\ Dept. of Computer Science \& Information Engineering \\ National Cheng Kung University \\ $\{$ klan, cmchou\}@csie.ncku.edu.tw
}

\begin{abstract}
A vehicular sensing network (VSN) is a type of wireless sensor network that empowers legacy resource-constrained WSNs by taking advantage of the rich network resources (e.g., the battery life, storage capability, and mobility) of vehicles. In this paper, we posit that the sensed data in VSNs is generally local in nature and propose a simple yet effective scheme, called To-and-Fro (TAF), for localized data dissemination. Unlike conventional wireless networks and sensing systems, the TAF scheme operates in an opportunistic manner and does not need a network infrastructure. More precisely, the scheme always forwards messages to passing vehicles if they are moving in the opposite direction. Using simulations of both synthetic and realistic network scenarios, we demonstrate that the proposed scheme is simple, practical, and effective for localized data dissemination in vehicular sensing networks. Moreover, the scheme is easy to deploy, as long as each vehicle in the network can access GPS information.
\end{abstract}

\section{Introduction}

A vehicular sensing network (VSN) is a type of wireless sensor network (WSN) that empowers legacy resourceconstrained WSNs by exploiting the rich resources (e.g., the battery life, storage capability, and mobility) of vehicles. In the recent years, a substantial amount of research effort has been invested in this area, and several real-world deployments have been carried out to demonstrate the feasibility and potential of VSNs. The potential applications of VSNs are wide ranging. For instance, it would be advantageous to employ vehicular sensing systems for reporting road surface conditions [8], monitoring environmental pollution [10], and providing urban surveillance [13].

VSNs differ from conventional wireless networks and sensing systems in a number ways. First, full-coverage cannot be guaranteed for infrastructure-based networks (e.g., WiMAX, GPRS, and 3G) in VSNs, since the latter may have to subsist in uninhabited areas and challenging environments (e.g., in mountains and desserts). Second, MANET-based solutions cannot be applied to VSNs directly, since network contacts (i.e., communication opportunities) are intermittent and unpredictable due to the diversity of vehicle mobility; and MANET-based solutions have difficulty maintaining end-to-end paths in a network. Third, delay tolerant network (DTN)-based solutions are not suitable either, as the sensed data in VSNs may vary/oscillate frequently, and DTN-based solutions are short on yield time and reliable data transmission. Moreover, in VSNs, the sensed data is mostly local in nature, i.e., vehicular networks are primarily interested in information from an event source that is nearby. For example, we may be interested in information about traffic jams on the route we are taking to a destination, or air pollution readings in the area where we work or live. Ideally, localized data dissemination should 1) only "affect" vehicles in the area where the information is relevant, and 2) ensure that all vehicles connected to the network in the area are aware of the information.

Intuitively, localized data dissemination in VSNs can be achieved by implementing geocasting-based approaches, which are designed to deliver messages to all interested peers within a geographical destination region. Although several data forwarding approaches have been proposed $[4,6,9,11,12,14,16,18]$, they are not specialized for VSN applications, and they do not consider the fact that, in VSNs, the geocast destination region is usually centered at the event source.

To address this research gap, we propose a novel solution, called To-and-Fro (TAF), for localized data dissemination in vehicular sensing networks. The TAF scheme is an opportunistic network-based approach that does not require a fully covered infrastructure-based network, and it is not necessary to maintain an end-to-end path between the source and the destination. Specifically, the TAF scheme follows a very simple rule: it only forwards messages to passing vehicles if they are traveling in the opposite direction. Using a comprehensive set of simulations, we evaluate the TAF scheme in terms of the spatial distribution, mes- 
sage population, dissemination ratio, and load distribution. The results show that the scheme is simple, practical, and very effective in preserving data sensed in the regions close to the event source, thereby fulfilling the goal of localized data dissemination. Moreover, it is easy to deploy, as long as the GPS information (i.e., the geographic position and the movement direction) can be accessed by each vehicle in the network.

The remainder of the paper is organized as follows. Section 2 contains a review of related works. In Section 3, we describe the proposed approach, called TAF, for localized data dissemination in opportunistic vehicular networks. Section 4 presents a comprehensive set of simulation results; the results are also analyzed and explained in detail. We then summarize our conclusions in Section 5.

\section{Related Work}

Localized data dissemination is a special case of geocasting, which is designed to deliver messages to nodes within a geographical region. The destination region of localized data dissemination is simply the area close to the message source. Geocasting approaches can be categorized into the following three types based on how messages are replicated and forwarded in the network: floodingbased approaches, directed flooding-based approaches, and non-flooding-based approaches [15]. Flooding-based approaches simply flood the network with messages (including information about the geocast destination region), and each receiver broadcasts the messages to its neighbors if it is within the specified destination region. The approaches are simple and robust; however, they are not efficient because the location information is not used to reduce the number of packets transmitted over the network.

Several directed flooding-based geocasting approaches have been proposed to avoid the tremendous traffic overhead caused by flooding-based approaches. For instance, Ko and Vaidya [11] proposed the Location Based Multicast (LBM) approach, which determines a forwarding zone for each message based on the carried geocast destination information, and only broadcasts the message to the forwarding zone. The approach was extended in [18], which applied the concept of Voronoi Diagrams to avoid the possible failures of the LBM approach (i.e., when the forwarding zone is empty or partitioned). In addition, the GeoGRID approach, proposed in [14], reduces the amount of redundant network traffic by partitioning the network into several logical grids and only allows cross-grid message transmission through the gateway nodes of the grids. In $[4,6]$, the authors propose creating a network mesh and only allow message transmission to take place on one network path in the mesh, even though there are multiple available paths.

Finally, non-flooding-based approaches use unicasting to

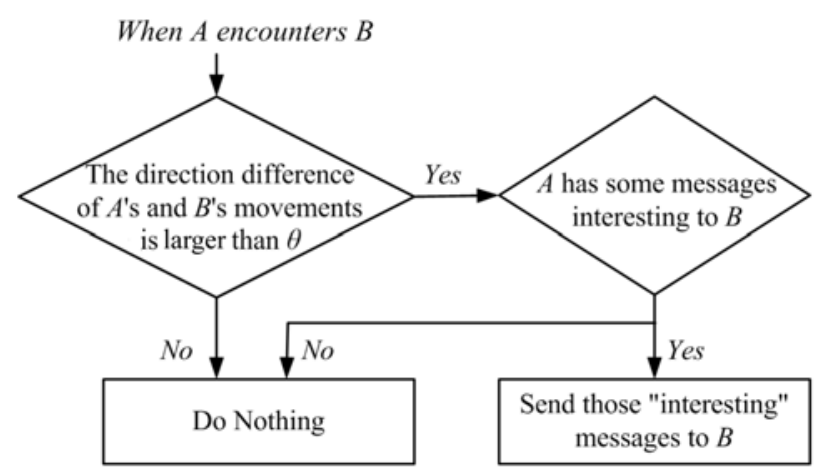

\section{Figure 1. The flowchart of the proposed local- ized data dissemination scheme.}

forward messages to the designated geocast destination regions. Once a message reaches its destination region, the receiver broadcasts the message to all of its neighbors within the region. For instance, in [16], Maihofer et al. propose using general unicast routing approaches to forward messages to the destination area. Each node on the unicast forwarding path checks whether it is in the geocast destination region. If it is in the destination region, it stops unicast forwarding and starts flooding the region with the message; otherwise, the node continues unicasting. Similarly, the GeoTORA approach [12] employs TORA unicast routing [17] to forward messages to the destination geocast group, and then broadcasts the messages to all members in the group. The GeoNode approach [9] utilizes a GPS-enabled infrastructure network and splits the data dissemination process into two phases: from the sender to its corresponding GeoNode (via unicasting), and from the GeoNode to the nodes inside the geocast destination region (via multiple unicasting or GPS-multicasting).

\section{The Proposed Approach}

In this section, we present the proposed localized message dissemination approach, called To-and-Fro (TAF). It exploits the mobility of VSNs, which are basically to-andfro-based. The objective of the TAF scheme is twofold: 1) to disseminate messages about local events in nearby areas only; and 2) to ensure that all vehicles in the nearby areas are aware of the local messages. In this study, for simplicity, we assume that each local event (e.g., a car accident) is witnessed by all passing vehicles, and is associated with a constant deadline $T_{d}$ (i.e., the event will be valid for $T_{d}$ and then expire). Figure 1 shows the flowchart of the To-andFro scheme.

More specifically, suppose $\overrightarrow{m_{A}}$ is the normalized movement vector of vehicle $A$, and $\pi(A, B)$ is the angle between the movement vectors of two vehicles $A$ and $B$. Then, 
$\pi(A, B)$ can be calculated by the arccos function of the inner product of $\overrightarrow{m_{A}}$ and $\overrightarrow{m_{B}}$, as shown in Eq. 1 .

$$
\pi(A, B)=\arccos \left(\overrightarrow{m_{A}} \cdot \overrightarrow{m_{B}}\right)
$$

When the TAF scheme is used in mobile vehicular sensing, there are two scenarios where a vehicle $A$ may encounter another vehicle $B$ on the road:

1. When $\pi(A, B) \geq \theta$ (i.e., $A$ and $B$ are moving in opposite directions), $A$ will forward to $B$ a number of messages that $B$ may be interested in.

2. When $\pi(A, B)<\theta$ (i.e., $A$ and $B$ are moving in the same direction), $A$ has no contact with $B$.

In these scenarios, $\theta$ is a constant threshold that determines 1) whether $A$ and $B$ are moving in opposite directions ${ }^{1}$; and 2) whether the messages that $B$ finds interesting are new to $B$ and have not expired yet.

\section{Evaluation}

To evaluate the performance of the proposed TAF scheme, we implemented it and performed simulations in DTNSim2 [1], a Java-based opportunistic network simulator. We assume there is only one source, which produces one new event every 60 seconds, and each event is valid for 300 seconds (i.e., $T_{d}=300$ ). Moreover, all vehicles passing the source witness the events that have been initiated and have not expired yet. We consider that two vehicles are moving in opposite directions if the angle of their movement vectors is greater than $90^{\circ}$ (i.e., $\theta=90^{\circ}$ ); otherwise, they are moving in the same direction.

We evaluate two network scenarios: one is a 1,600 $\times$ 1,600 meter area, and the other is a $900 \times 900$ meter area. The former scenario is based on a $5 \times 5$ grid topology, and the latter was created by TIGER [3] based on a map of the Afton Oaks area in Houston, Texas, as shown in Figure 2. We assume the event source is located at the center of the network topology (indicated by the red point), and there are 12 vehicle flows in the first scenario and 8 flows in the second one (indicated by the blue arrows). Moreover, we set the inter-arrival time of the vehicles per flow as a constant $T_{a}$, and assume that each vehicle leaves the area after it reaches the map boundary. The vehicular traffic is then generated by a microscopic, space continuous and time discrete traffic simulation tool called SUMO [2]. A network contact (i.e., a communication opportunity between two vehicles) occurs if two vehicles are within each other's wireless transmission range, which is set to $100 \mathrm{~m}$ in this study. Table 1 details the properties of the two network scenarios,

\footnotetext{
${ }^{1}$ For simplicity, we set $\theta$ to $90^{\circ}$, and defer a detailed discussion and evaluation of this issue to a future work.
}

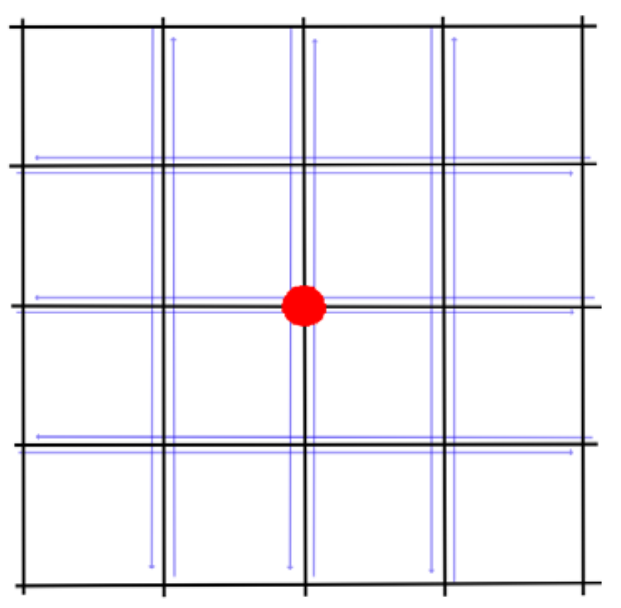

(a) Scenario 1

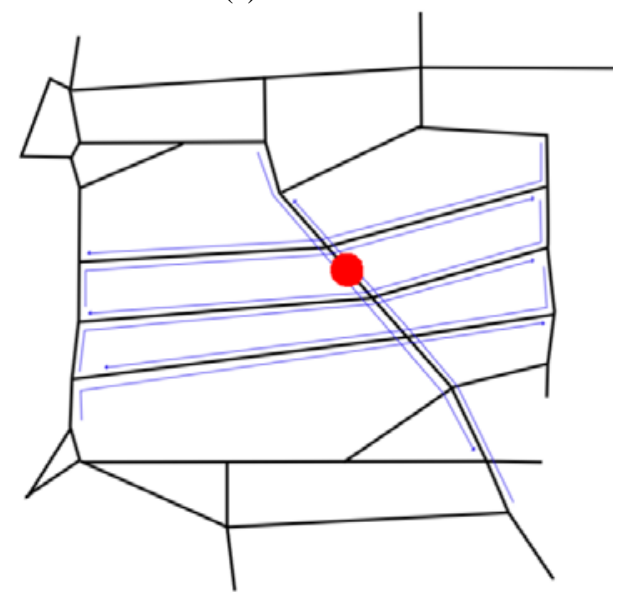

(b) Scenario 2

Figure 2. The topology of the evaluated network scenarios: (a) a $5 \times 5$ grid, and (b) a map of the Afton Oaks area in Houston, Texas.

and Figure 3 shows the CDF distribution of the number of network contacts made by each vehicle.

For simplicity, we assume that each vehicle has an infinite buffer size, and the wireless data transmission between vehicles is error free with a fixed bandwidth of 5Mbps. All the presented results are based on the average performance of 200 simulation runs for each network configuration.

\subsection{Evaluation I: Spatial Distribution}

In the first set of simulations, we evaluate the spatial distribution (i.e., the dissemination distance) of the proposed TAF scheme over each message's lifetime in the two network scenarios. The dissemination distance is the average distance between the event source and the vehicles that are aware of it (i.e., vehicles that carry a message about 


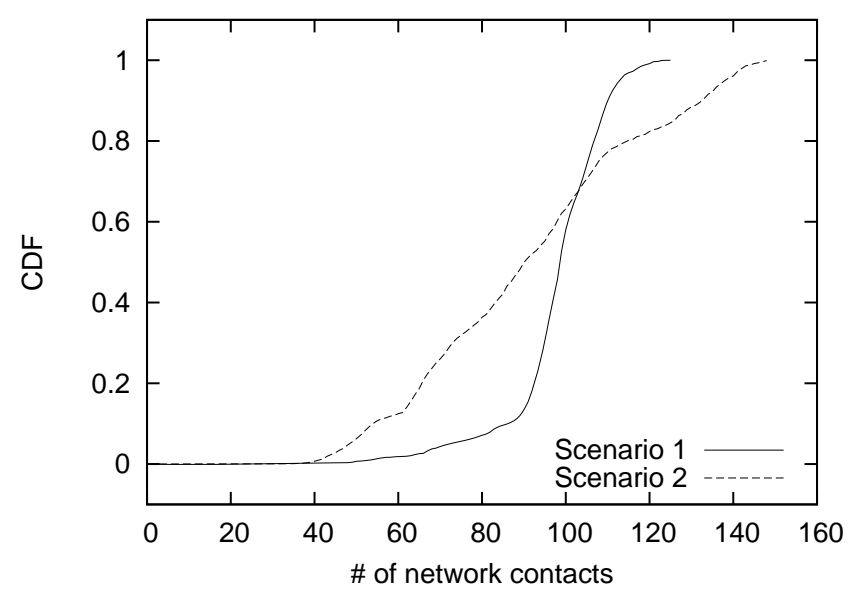

Figure 3. The distribution of the number of network contacts made by each vehicle in the described network scenarios.

\section{Table 1. The properties of the two network scenarios}

\begin{tabular}{c|cc} 
& Scenario 1 & Scenario 2 \\
\hline Duration (seconds) & 2,500 & 2,500 \\
Size (meters) & $1,600 \times 1,600$ & $900 \times 900$ \\
\# of vehicles & 1,800 & 1,680 \\
\# of vehicle flows & 12 & 8 \\
inter-arrival time $T_{a}(\mathrm{~seconds})$ & 14 & 10 \\
Avg speed $(\mathrm{km} / \mathrm{hr})$ & 20.0839 & 25.6898 \\
Max speed $(\mathrm{km} / \mathrm{hr})$ & 49.9985 & 79.4455 \\
Number of contacts & 173,330 & 139,558 \\
Avg \# Contacts/pair & 0.107053 & 0.098952
\end{tabular}

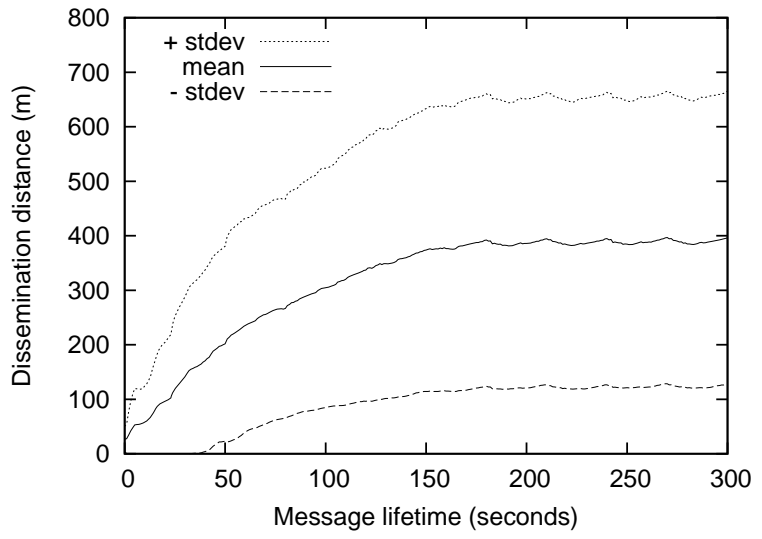

(a) Scenario 1

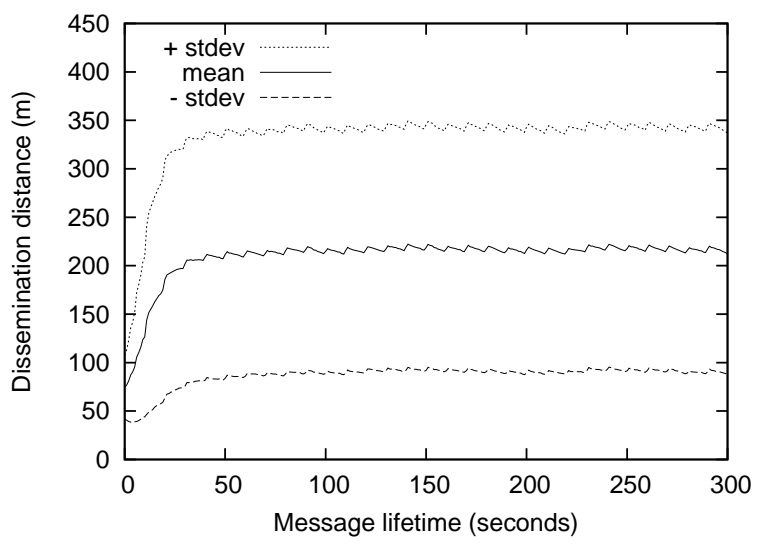

(b) Scenario 2

Figure 4. The average distance of message dissemination (with a standard deviation of \pm 1 ) over time. the event). This is a good evaluation metric for indicating whether the TAF scheme can efficiently disseminate messages in local areas.

From the simulation results, shown in Figure 4, we observe that there are two phases in the curve of the average dissemination distance: a linearly increasing phase, and a convergence phase. For instance, in the first network scenario, the average distance increases linearly in the first 160 seconds, and then converges to 390 meters; whereas, in the second network scenario, the average distance increases linearly in the first 30 seconds, and then converges to $210 \mathrm{me}-$ ters.

Specifically, in the linearly increasing phase, because the event has only just occurred, only a small number of vehicles are aware of it, and most of them witness the event independently (i.e., rather than learning about it from other vehicles). Since these 'early witnesses' are moving away from the source, the average dissemination distance increases linearly over time. Meanwhile, in the convergence phase, a comparable number of vehicles, which are moving towards the source, learn about the event from the 'early witnesses' as they pass each other. As a result, the average dissemination distance stops increasing and starts to converge to some point.

In the second network scenario, the linearly increasing phase is much shorter than that of the first scenario, since the distance between the event source and its closest intersection is much shorter than in the first scenario. Therefore, the early witnesses have more opportunities to forward stored messages to the passing vehicles in the second network scenario, which reduces the length of the linearly increasing phase. 


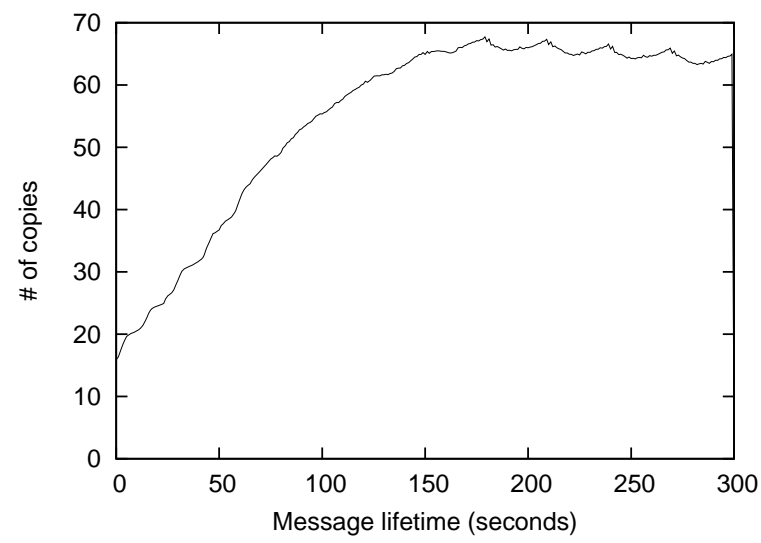

(a) Scenario 1

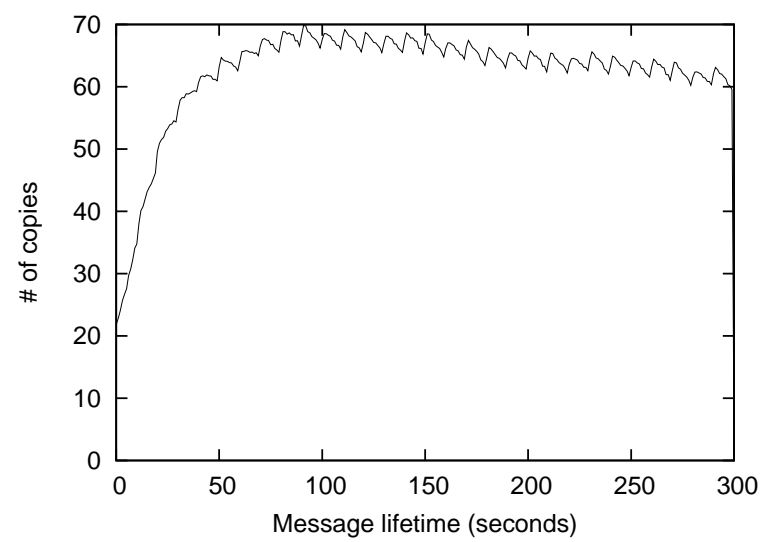

(b) Scenario 2

Figure 5. The average number of message copies per event in the network.

\subsection{Evaluation II: Message Population}

Next, we evaluate the distribution of the message population under the TAF scheme in the two network scenarios. The message population is the average number of messages about each event existing in the network (i.e., the average number of vehicles that are aware of a particular event). Generally, the larger the message population, the more resilient the dissemination scheme will be against malicious network attacks $[5,7]$.

Figure 5 shows the simulation results. Similar to the spatial distribution, the curves increase almost linearly initially, and then converge to some point with slight fluctuations. Here, the linearly increasing phase is caused by the fact that the number of witnesses (either firsthand or secondhand) increases as the lifetime of the event increases. However, after some period, as the number of vehicles leaving the network increases (due to the size limitation of the network scenarios), the curve stops increasing and enters the convergence phase.

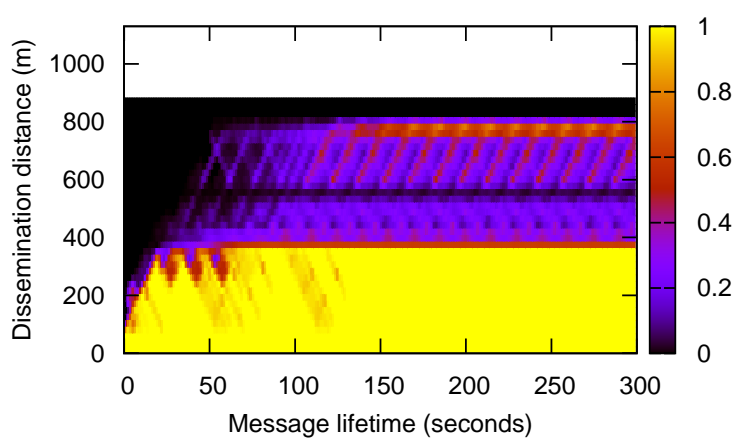

(a) Scenario 1

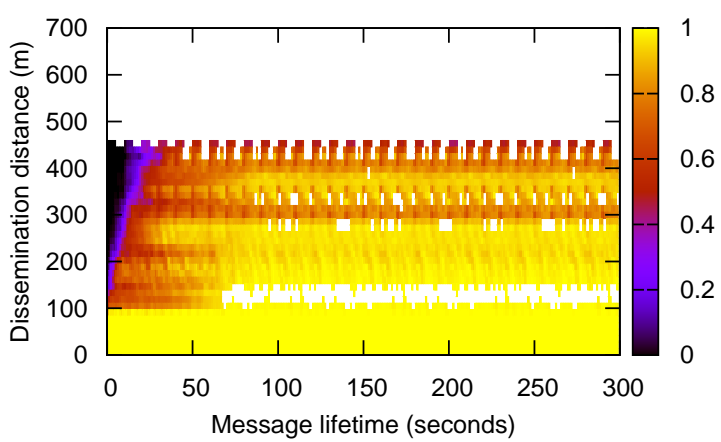

(b) Scenario 2

Figure 6. The percentage of affected vehicles within a specific distance of the event source.

The linearly increasing phase in the second scenario is shorter than that in the first scenario. This is because the second network scenario is smaller $(900 \mathrm{~m} \times 900 \mathrm{~m})$ than the first network scenario $(1,600 \mathrm{~m} \times 1,600 \mathrm{~m})$. As a result, vehicles leave the second network more quickly, which results in a shorter linearly increasing phase.

\subsection{Evaluation III: Dissemination Ratio}

Here, we evaluate the dissemination ratio of the proposed TAF scheme, i.e., the proportion of nodes that are aware of a particular event and are within a certain distance of it. Intuitively, the dissemination ratio should decrease as the distance from the event increases. Moreover, the ideal localized data dissemination solution should achieve a high dissemination ratio, especially in the area close to the event. Figure 6 shows the simulation results.

The results in Figure 6 demonstrate that, during the lifetime of a message, the dissemination ratio decreases as the distance from the event increases; and, for any distance, 


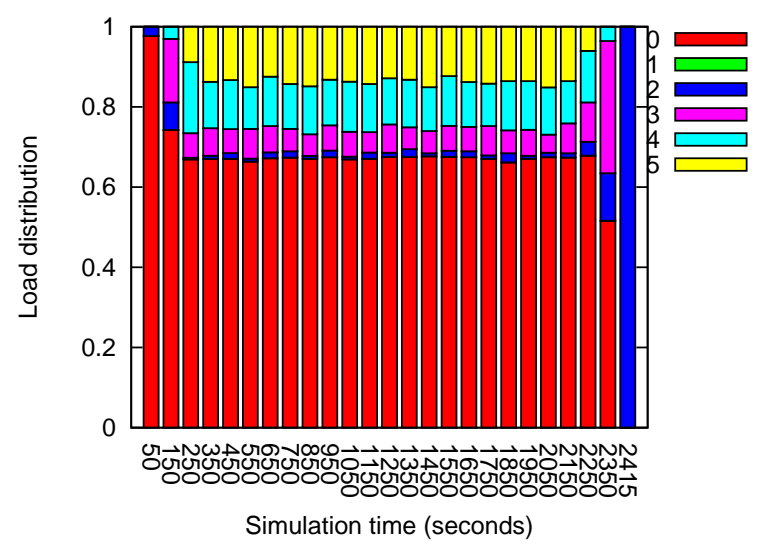

(a) Scenario 1

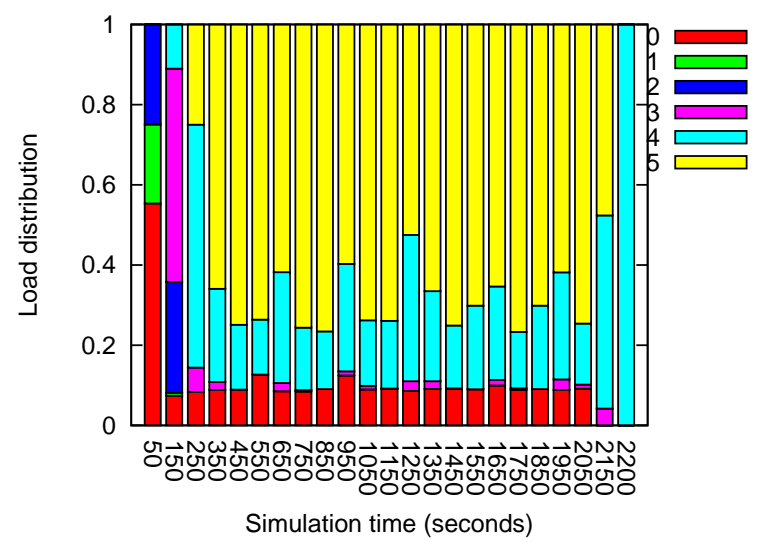

(b) Scenario 2

\section{Figure 7. The load distribution of the vehicles in the network.}

the dissemination ratio increases as the message lifetime increases (i.e., an increasing proportion of nearby nodes become aware of the event over time). The results confirm our intuition that the dissemination ratio increases over time and decreases as the distance increases. Moreover, the dissemination ratio in the second network scenario is higher than that in the first network scenario. Specifically, in the second network scenario, when the message lifetime is $100 \mathrm{sec}-$ onds, the dissemination ratio is greater than 0.5 when the distance is less than 400 meters; whereas the ratio fluctuates a great deal in the range 0.2 to 1 in the first network scenario. This is because the vehicular traffic in the first scenario (i.e., the grid topology) is too regular to provide sufficient network dynamics for the TAF scheme. Fortunately, this problem hardly occurs in reality because of the diverse mobility of vehicular traffic.

\subsection{Evaluation IV: Load Distribution}

In the last set of simulations, we evaluate the load distribution of the TAF scheme, i.e., the distribution of the number of messages carried per vehicle in the network. Intuitively, the higher the load distribution (i.e., the more duplicates of the message that exist in the network), the more resilient the data dissemination scheme should be. However, on the downside, if the data dissemination scheme has a higher load distribution, it consumes more storage space on each vehicle. Thus, it is more likely to encounter the buffer overflow problem, especially when the vehicular network is very dense and/or the number of simultaneous events is very large.

Figure 7 shows the simulation results of the proposed scheme for both network scenarios. Note that, since the event source generates a new event every 60 seconds in our simulations and each event is only valid for 300 seconds, the maximum number of simultaneously valid messages is $300 / 60=5$. The results show that, in the first network scenario, the buffer consumption of the proposed scheme is about 1.14 messages; approximately $10 \%$ of the vehicles carry 5 messages, $10 \%$ carry 4 messages, $6 \%$ carry 3 messages, $3 \%$ carry 2 messages and $71 \%$ carry 1 message or no message. In the second network scenario, the buffer consumption is about 3.9 messages; approximately $50 \%$ of the vehicles carry 5 messages, $25 \%$ carry 4 messages, $10 \%$ carry 3 messages, $4 \%$ carry 2 messages, and $11 \%$ carry 1 message or no message.

The results indicate two facts. First, the load distribution of the proposed TAF scheme is moderate because its "buffer consumption" is about $1.14 / 5=22.8 \%$ and $3.9 / 5=78 \%$ of the concurrent valid messages in the two network scenarios respectively (i.e., the vehicles are not forced to listen to messages that are not nearby). Second, the load distribution tends to become more even if the vehicles stay longer in the area close to the event source (i.e., the network topology is more like a "closed loop"). For instance, the load distribution is much more even in the second network scenario than in the first network scenario. This is because the average distance from the event source to the boundary of the map is larger in the second scenario than in the first scenario (i.e., the vehicles generally leave the map more quickly in the first scenario).

\section{Conclusion}

In this paper, we study localized data dissemination in vehicular sensing networks, and propose a simple yet effective approach, called To-and-Fro (TAF), for such data dissemination. Unlike conventional geocast-based approaches, which are designed for generic mobile ad hoc networks (MANETs), the TAF scheme is tailored for opportunis- 
tic vehicular networks in which intermittent connectivity is common and network partitioning may occur. Using a comprehensive set of simulations, we evaluate the scheme in terms of the spatial distribution, message population, dissemination ratio, and load distribution. The results show that TAF is effective for localized data dissemination, i.e., it stores live information in the area close to the event. Work on reducing the communication overhead and tuning the system parameters is ongoing. We hope to report the results in the near future.

\section{Acknowledgement}

This research was supported by the National Science Council of Taiwan under grant number NSC 97-2628-E001-007-MY3.

\section{References}

[1] DTNSim2: A Delay-Tolerant Network Simulator. http://watwire.uwaterloo.ca/DTN/sim/.

[2] Simulation of Urban MObility (SUMO): an open source traffic simulation package. http://sumo.sourceforge.net/.

[3] Topologically Integrated Geographic Encoding and Referencing system (TIGER). http://www.census.gov/geo/www/tiger/.

[4] J. Boleng, T. Camp, and V. Tolety. Mesh-based geocast routing protocols in an ad hoc network. In International Parallel \& Distributed Processing Symposium, 2001.

[5] J. Burgess, G. D. Bissias, M. Corner, and B. N. Levine. Surviving attacks on disruption-tolerant networks without authentication. In ACM MobiHoc, 2007.

[6] T. Camp and Y. Liu. An adaptive mesh-based protocol for geocast routing. Journal of Parallel and Distributed Computing, 63(2):196-213, February 2003.

[7] L.-J. Chen, C.-L. Chiou, and Y.-C. Chen. An evaluation of routing reliability in non-collaborative opportunistic networks. In IEEE International Conference on Advanced Information Networking and Applications, 2009.

[8] J. Eriksson, L. Girod, B. Hull, R. Newton, S. Madden, and H. Balakrishnan. The pothole patrol: Using a mobile sensor network for road surface monitoring. In ACM International conference on Mobile Systems, Applications and Services, 2008.
[9] T. Imielinski and J. Navas. GPS-Based Addressing and Routing. IETF RFC 2009, November 1996.

[10] E. Kanjo and P. Landshoff. Fresh: Cellid based mobile forum for community environmental awareness. In Workshop on Ubiquitous Sustainability: Citizen Science \& Activism, 2008.

[11] Y.-B. Ko and N. H. Vaidya. Geocasting in mobile ad hoc networks: Location-based multicast algorithms. In IEEE Workshop on Mobile Computer Systems and Applications, 1999.

[12] Y.-B. Ko and N. H. Vaidya. Anycasting-Based Protocol for Geocast Service in Mobile Ad Hoc Networks. Computer Networks, 41(6):743-760, April 2003.

[13] U. Lee, E. Magistretti, M. Gerla, P. Bellavista, and A. Corradi. Dissemination and Harvesting of Urban Data Using Vehicular Sensing Platforms. IEEE Transactions on Vehicular Networks, To Appear.

[14] W.-H. Liao, Y.-C. Tseng, K.-L. Lo, and J.-P. Sh. GeoGRID: A Geocasting Protocol for Mobile Ad Hoc Networks Based on GRID. Journal of Internet Technology, 1(2):23-32, December 2000.

[15] C. Maihofer. A Survey of Geocast Routing Protocols. IEEE Communications Surveys \& Tutorials, 6(2):3242, 2004.

[16] C. Maihofer, W. Franz, and R. Eberhardt. Stored Geocast. In Proceedings of Kommunikation in Verteilten Systemen, 2003.

[17] V. D. Park and M. S. Corson. A Highly Adaptive Distributed Routing Algorithm for Mobile Wireless Networks. In IEEE Infocom, 1997.

[18] I. Stojmenovic, P. Ruhil, and D. K. Lobiyal. Voronoi Diagram and Convex Hull-Based Geocasting and Routing in Wireless Networks. In IEEE Symposium on Computer and Communication, 2001. 